\title{
Vibration Characteristic Analysis of a Cavity Treated with Passive Constrained Layer Damping
}

\author{
LU Jing ${ }^{1, a,}$, WANG Yu-Xiang ${ }^{2, b}$, YUAN Li-yun ${ }^{3, c}$ \\ ${ }^{1}$ College of Automobile and Transportation, Guangxi University of Science and Technology, Liu Zhou, \\ 545006, China \\ ${ }^{2}$ College of Automobile and Transportation, Guangxi University of Science and Technology, Liu Zhou, \\ 545006, China \\ ${ }^{3}$ College of Automobile and Transportation, Guangxi University of Science and Technology, Liu Zhou, \\ 545006, China \\ axxustlj@126.com ,bfaxiaoke@163.com , cflowlily@126.com \\ * Corresponding author
}

Keywords: Passive constrained layer damping, cavity, dynamic model, vibration characteristic

\begin{abstract}
A semi-analytical method was proposed to analyze the dynamics characteristics of a cavity treated with passive constrained layer damping (PCLD). Based on the laminated theory, the dynamics equations of a PCLD beam was derived firstly. Then the transfer matrix between beams was developed according to the displacement continuity conditions and equilibrium equations of the junction. Combing the state equations of the beams, the transfer matrix between beams, and the boundary conditions, the dynamic model of a PCLD cavity was established in this paper. The comparisons with the results of FEM and document have certificated the correction of the present model. The numerical calculations show that the PCLD treatment can damp the vibration well, especially in the higher frequency, and the damping effect increases with the increasing of the thickness of the viscoelastic, but it may be decrease when the thickness of the constrained increases.
\end{abstract}

\section{Introduction}

In the aerospace, transportation and other engineering fields, many structures exist elastic cavity, such as the aircraft cabin, car cars, etc. These structures can be simplified to a closed cavity with a certain shape. Under the action of external and sound source, the elastic cavity structure will vibration and cause a lot of internal noise in its internal space, which will not only reduce the ride comfort, but also affect the normal work of the internal equipment, as well as the life and safety of the structure. Therefore, the cavity vibration characteristic is one of the important indicators in thin-walled cavity structure design, it is also the basis for the further cavity acoustic properties analysis ${ }^{[1]}$. Passive Constrained Layer Damping (PCLD) has gradually replaced the free damping, which is widely used in structural vibration and noise reduction ${ }^{[2]}$. At present, the analysis of PCLD structure usually adopts finite element method ${ }^{[3]}$. However, due to the existence of meshing, the calculation accuracy and efficiency of the finite element method will decrease with the increase of frequency. In this paper, the kinetic model of the PCLD cavity was established by using the dynamic equation of PCLD beam, the displacement continuity condition and mechanical equilibrium equation at junction point. The accuracy of the model and the calculation method is proved by comparing with the literature value and the finite element results. By using this method, the dynamic response of the cavity structure was calculated and the influence of structural parameters on the vibration characteristics and damping characteristics of the cavity structure was also analyzed. 


\section{Dynamic Equations of PCLD Beam}

The PLCD beam is usually composed of a base beam, viscoelastic layer (VEM) and constrained layer (CL), denoted by 1, 2 and 3 in the paper. In order to facilitate the derivation, we use the following assumptions ${ }^{[4]}$ : (1) the horizontal displacement of a point is the same in each layer; (2) the width of each layer is same; (3) comparing with the base beam and CL, the Young's modulus of the VEM can be neglected, so only the shearing deformation is concerned in the VEM; (4) the layers are connected ideally, and there is not relative slip; (5) the layers meet the linear theory.

Under the action of harmonic excitation, the normal equilibrium equation of VEM can be written as

$$
p_{z}^{(12)}-p_{z}^{(23)}+\rho_{2} b h_{2} \omega^{2} w=0,
$$

in which, $p_{z}^{(12)}, p_{z}^{(23)}$ denote the transverse force amplitude of the base beam and $C L$ acting on the $V E M, \rho_{2}$ the density of the viscoelastic layer, $b$ the width of the beam, $h_{2}$ the thickness of the viscoelastic layer, $\omega$ the circular frequency of the excitation, $w$ the transverse displacement of the beam.

Considering the influence of the shear deformation and the eccentric moment of the VEM, the tangential forces applied to the beam and CL are expressed as follows

$$
\begin{aligned}
& p_{x}^{(1)}=f_{x}^{(1)}+\tau_{z x}, p_{z}^{(1)}=f_{z}^{(1)}-\tilde{p}_{z}^{(12)}+f_{e}^{(1)} \\
& p_{x}^{(3)}=f_{x}^{(3)}-\tau_{z x}, p_{z}^{(3)}=f_{z}^{(3)}+\tilde{p}_{z}^{(23)}+f_{e}^{(3)},
\end{aligned}
$$

in which, $f_{x}^{(1)}$ and $f_{x}^{(3)}$ are the axial load acted on the base beam and CL respectively, $f_{z}^{(1)}$ and $f_{z}^{(3)}$ the transverse load acted on the base beam and CL respectively and $\tau_{z x}$ the shear stress of the viscoelastic layer neutral layer.

According to the bending theory, we can obtain the first order ordinary differential control equation of the base beam and CL

$$
\begin{aligned}
& \frac{d \overline{\boldsymbol{Y}}_{1}}{d \xi}=\boldsymbol{G}_{1} \overline{\boldsymbol{Y}}_{1}+\overline{\boldsymbol{P}}_{1} \\
& \frac{d \overline{\boldsymbol{Y}}_{3}}{d \xi}=\boldsymbol{G}_{3} \overline{\boldsymbol{Y}}_{3}+\overline{\boldsymbol{P}}_{3},
\end{aligned}
$$

in which, $\overline{\boldsymbol{Y}}=\left\{\bar{u}, \bar{w}, \bar{\phi}_{x}, \bar{N}_{x}, \bar{Q}_{x}, \bar{M}_{x}\right\}^{T}$ is the state vector, $\overline{\boldsymbol{P}}=-\frac{L}{E A}\left\{\begin{array}{llllll}0 & 0 & 0 & p_{x} & p_{z} & 0\end{array}\right\}^{T}$ the load vector, where $x=\xi L, L$ is any length of dimensionless; $u=L \bar{u} 、 w=L \bar{w} 、 \phi_{x}=\bar{\phi}_{x}$ are the amplitudes of the axial displacement, transverse displacement and rotation of the neutral layer; $N_{x}=(E A) \bar{N}_{x} 、 Q_{x}=(E A) \bar{Q}_{x} 、 M_{x}=(E I / L) \bar{M}_{x}$ are the amplitudes of the axial force, shear force and the moment of bending; $A$ is the cross-sectional area of the beam, and $E$ is the Young's modulus of the material.

According to the displacement continuity condition of the interlayer, the shear stress $\mathrm{X}$ of the neutral layer of the viscoelastic layer can be expressed by the displacement of the base beam and the confinement layer by the first-order shear deformation theory and Hooke's law, replace it with (2), and combined with (1) (4), The derivative control equation of PCLD beam can be deduced.

$$
\frac{d \overline{\mathbf{Z}}}{d \xi}=\overline{\mathbf{B}} \overline{\mathbf{Z}}+\overline{\boldsymbol{F}},
$$

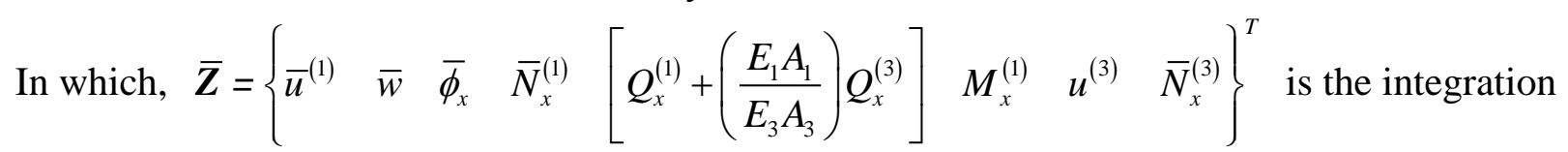
state vector, $\overline{\boldsymbol{F}}=-\left(\frac{L}{E_{1} A_{1}}\right)\left\{\begin{array}{lllllll}0 & 0 & 0 & f_{x}^{(1)} & \left(f_{z}^{(1)}+f_{z}^{(3)}\right) & 0 & 0\end{array}\left(\frac{E_{1} A_{1}}{E_{3} A_{3}}\right) f_{x}^{(3)}\right\}^{T}$ is integrated load 
vector. Nonzero elements in the $\bar{B}$ matrix are: $\bar{B}(1,4)=1 ; \bar{B}(2,3)=-1 ; \quad \bar{B}(3,6)=1$;

$\bar{B}(4,1)=-\lambda_{1}^{2}-\left(\frac{L S_{1} b}{E_{1} A_{1}}\right), \bar{B}(4,3)=-\left(\frac{L S_{2} b}{E_{1} A_{1}}\right), \bar{B}(4,7)=-\left(\frac{L S_{3} b}{E_{1} A_{1}}\right) ; \quad \bar{B}(5,2)=-\lambda_{1}^{2}-\left(\frac{E_{3} A_{3}}{E_{1} A_{1}}\right) \lambda_{3}^{2}-\lambda_{2}^{2}$,
$\bar{B}(5,4)=-\left(\frac{e S_{1} b}{E_{1} A_{1}}\right), \bar{B}(5,6)=-\left(\frac{e S_{2} b}{E_{1} A_{1}}\right), \bar{B}(5,8)=-\left(\frac{e S_{3} b}{E_{1} A_{1}}\right) ; \bar{B}(6,5)=\frac{L^{2} A_{1}}{I_{1}\left[1+\left(\frac{E_{3} I_{3}}{E_{1} I_{1}}\right)\right]} \bar{B}(7,8)=1 ;$

$\bar{B}(8,1)=\left(\frac{L S_{1} b}{E_{3} A_{3}}\right), \bar{B}(8,3)=\left(\frac{L S_{2} b}{E_{3} A_{3}}\right), \bar{B}(8,7)=\left[-\lambda_{3}^{2}+\left(\frac{L S_{3} b}{E_{3} A_{3}}\right)\right]$.In the elements, constant

$e=e_{1}+e_{3}, e_{1}, e_{3}$ which are the eccentricity of the neutral layer of the viscoelastic layer to the base layer and the neutral layer of the confinement layer, respectively; the constant $S_{1}=-\frac{G_{2} L}{h_{2}}, S_{2}=-G_{2}\left(\frac{h_{3}+h_{1}}{2 h_{2}}+1\right), S_{3}=\frac{G_{2} L}{h_{2}}, G_{2}$ Indicates the thickness of the viscoelastic layer; the frequency factor $\lambda_{2}^{2}=\frac{\rho_{2} h_{2} L^{2} b \omega^{2}}{E_{1} A_{1}}$.

\section{Dynamic Model of PCLD Cavity}

Laying PCLD cavity can be regarded as a number of flexible beam and PCLD beam connected, as shown in Figure 1. The control equation of the single-layer elastic beam is Eq.(3), and the control equation of PCLD beam is Eq.(5). The formula Eq. (3) and Eq.(5) are obtained by homogeneous expansion and precise integration method ${ }^{[5]}$.The relationship between the starting point of the beam and the end state vector

$$
\begin{gathered}
\overline{\boldsymbol{Y}}_{i 2}=\boldsymbol{M}_{i} \overline{\boldsymbol{Y}}_{i 1}+\boldsymbol{I}_{i} \\
\overline{\boldsymbol{Z}}_{j 2}=\boldsymbol{N}_{j} \overline{\boldsymbol{Z}}_{j 1}+\boldsymbol{J}_{j},
\end{gathered}
$$

in which , the subscripts $I, j$ denote the number of the single-layer beam and PCLD beam; 1,2 the starting and ending points of the beam; $M, N$ the transfer matrix of the single-layer beam and the PCLD beam; $I$, $J$ the non - homogeneous term caused by the load vector.

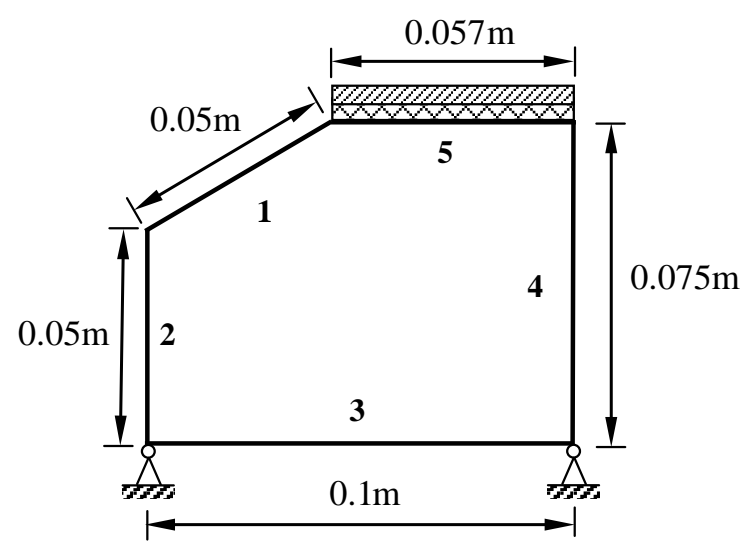

Fig.1 A cavity treated with passive constrained layer damp

At the connection point between the ending point of the $I$ beam and the starting point of the $i+1$ beam, the displacement continuity condition can be written as 


$$
\begin{aligned}
& u_{(i+1) 1}=u_{i 2} \cos \alpha-w_{i 2} \sin \alpha \\
& w_{(i+1) 1}=u_{i 2} \sin \alpha+w_{i 2} \cos \alpha \\
& \phi_{x(i+1) 1}=\phi_{x i 2},
\end{aligned}
$$

in which , $\alpha$ is the angle between the $I$ beam and the $i+1$ beam.

At the connection point between the ending point of the $I$ beam and the starting point of the $i+1$ beam, the equilibrium equation of forces can be written as

$$
\begin{aligned}
& N_{x(i+1) 1}-N_{x i 2} \cos \alpha+Q_{x i 2} \sin \alpha-F_{x k} \cos \alpha-F_{y k} \sin \alpha=0 \\
& Q_{x(i+1) 1}-N_{x i 2} \sin \alpha-Q_{x i 2} \cos \alpha-F_{x k} \sin \alpha+F_{y k} \cos \alpha=0 \\
& M_{x(i+1) 1}-M_{x i 2}+M_{k}=0,
\end{aligned}
$$

in which , $F_{x}, F_{y}, M$ represents the external force acting at the connection point, and the subscript $k$ represents the number of the external load.

The continuity conditions of the ending point of the $I$ beam and the starting point of the $i+1$ beam are obtained from (8) and (9)

$$
\left[\begin{array}{ccc}
6 \times 6 & 6 \times 6 & 6 \times 3 \\
\boldsymbol{T}_{1} & \boldsymbol{T}_{2} & \boldsymbol{T}_{3}
\end{array}\right]\left\{\begin{array}{c}
\overline{\boldsymbol{Y}}_{i 2} \\
\overline{\boldsymbol{Y}}_{(i+1) 1} \\
\overline{\boldsymbol{R}}_{k}
\end{array}\right\}=0,
$$

In which, $\overline{\boldsymbol{R}}_{k}=\left\{\bar{F}_{x k}, \bar{F}_{y k}, \bar{M}_{k}\right\}^{T}$ is the force vector acting on the connection point. The nonzero elements in the coefficient matrix $\boldsymbol{T}_{1}$ are:

$T_{1}(1,2)=\sin \alpha, T_{1}(2,1)=-\sin \alpha, T_{1}(2,2)=-\cos \alpha, T_{1}(3,3)=-1, \quad T_{1}(4,4)=-\cos \alpha, \quad T_{1}(4,5)=\sin \alpha$, $T_{1}(5,4)=-\sin \alpha, T_{1}(5,5)=-\cos \alpha, T_{1}(6,6)=-1$. The coefficient matrix $\boldsymbol{T}_{2}$ is a 6-order unit matrix, and the non-zero elements in the coefficient matrix $\boldsymbol{T}_{3}$ are: $T_{3}(4,13)=-\cos \alpha$, $T_{3}(4,14)=-\sin \alpha, \quad T_{3}(5,13)=-\sin \alpha, \quad T_{3}(5,14)=\cos \alpha, T_{3}(6,15)=1$.

the cavity be composed of $m$ elastic beams and $n$ PCLD beams, $6 m+8 n$ algebraic equations can be obtained by Eq.(6) and Eq.(7). There are $m+n$ connection points in the PCLD cavity. Then $6(m+n)$ algebraic equations can be obtained by Eq.(10). The two ends of the CL are free constrain in each PCLD beam, that is $N_{x}^{(3)}=0$, so we can obtain $2 n$ algebraic equations. To sum up, $12 m+16 n$ algebraic equations can be given. Each beam has two state vectors, so the number of components of the cavity state vector are also $12 m+16 n$. On the other hand, since the boundary condition and the unknown vector are dual, as there are three fixed boundary conditions ( $u=w=\phi_{x}=0$ ), there are three components of the unknown state vector $\left(F_{x}, F_{y}, M\right)$ too. Thus, the number of cavity boundary conditions is also equal to the number of additional components. The above state equation, continuity conditions, boundary conditions are assembled into a matrix form

$$
\boldsymbol{H A}=\boldsymbol{K},
$$

in which , $l$ is the number of additional external force vectors, the coefficient matrix $H$ is the $12 m+16 n+l$ order matrix, $A$ is the $12 m+16 n+l$ order vector, $A=\left\{\begin{array}{llllllllllllllllll}\overline{\boldsymbol{Y}}_{11} & \overline{\boldsymbol{Y}}_{12} & \overline{\boldsymbol{Y}}_{21} & \overline{\boldsymbol{Y}}_{22} & \cdots & \overline{\boldsymbol{Y}}_{m 1} & \overline{\boldsymbol{Y}}_{m 2} & \overline{\boldsymbol{Z}}_{11} & \overline{\mathbf{Z}}_{12} & \overline{\mathbf{Z}}_{21} & \overline{\mathbf{Z}}_{22} & \cdots & \overline{\boldsymbol{Z}}_{m 1} & \overline{\boldsymbol{Z}}_{m 2} & \boldsymbol{R}_{1} & \boldsymbol{R}_{2} & \cdots & \left.\boldsymbol{R}_{l}\right\}^{T} \boldsymbol{K}\end{array}\right.$ is a nonhomogeneous state vector of $12 m+16 m+l$ order.

The components of the state vector can be solved by Eq. (11). Taking these components as the initial value, and using the homogeneous expansion precise integral method once again, one can analyze the vibration characteristics of any point on the cavity. 


\section{Numerical Examples}

Example 1 In order to verify the accuracy of the dynamic model of PCLD beam, consider a beam with full PCLD treatment. The length of the beam is $L=0.1 \mathrm{~m}$, the height of each layer is $h_{1}=0.008 \mathrm{~m}, h_{2}=0.005 \mathrm{~m}, h_{3}=0.003 \mathrm{~m}$. The width of the beam is $b=0.005 \mathrm{~m}$. The elastic modulus of the beam and CL are $E_{1}=E_{3}=206 \mathrm{GPa}$, the densities are $\rho_{1}=\rho_{3}=7800 \mathrm{~kg} / \mathrm{m}^{3}$. The density of the VEM is $\rho_{2}=2600 \mathrm{~kg} / \mathrm{m}^{3}$. The shear modulus is $G_{2}=9.8(1+0.1 \mathrm{i}) \mathrm{GPa}$. The natural frequency and loss factor of the beam are calculated, and the comparison with the document results are shown in Table 1 . In the table, the natural frequency and the loss factor are defined as $f=\operatorname{Re}(\omega) / 2 \pi$, $\eta=2 \operatorname{Im}(\omega) / \sqrt{[\operatorname{Im}(\omega)]^{2}+[\operatorname{Re}(\omega)]^{2}}$.

Table 1 Comparisons of the nature frequency and loss factor of the PCLD beam with the document results

\begin{tabular}{ccccc}
\hline & \multicolumn{2}{c}{ Natural frequency $(\mathrm{Hz})$} & \multicolumn{2}{c}{ Loss factor } \\
\cline { 2 - 5 } order & document[4] data & present data & document[4] data & present data \\
\hline 1 & 3514 & 3502 & 0.0149 & 0.0144 \\
2 & 11148 & 11759 & 0.0349 & 0.0329 \\
3 & 22546 & 22547 & 0.0401 & 0.0401 \\
\hline
\end{tabular}

It can be seen from the table that, the present method is in good agreement with the document results, which proves the accuracy of the present model.

Example 2 In order to verify the correctness of modeling method of the cavity structure, considering a flexible cavity, the size and shape are the same as Fig.1, and each beam is a single-layer elastic beam with a height $h=0.008 \mathrm{~m}$, width $b=0.005 \mathrm{~m}$, elastic modulus $E=206 \mathrm{GPa}$, density $\rho=7800 \mathrm{~kg} / \mathrm{m}^{3}$.Using finite element method to calculate the natural frequency of the cavity. The results are compared with the present method and shown in Fig.2. Fig. 3 is the second-order vibration diagram calculated by the finite element software. It can be seen from the figure that the present results are very close to those of the finite element method, and the calculated error is less than $5 \%$.

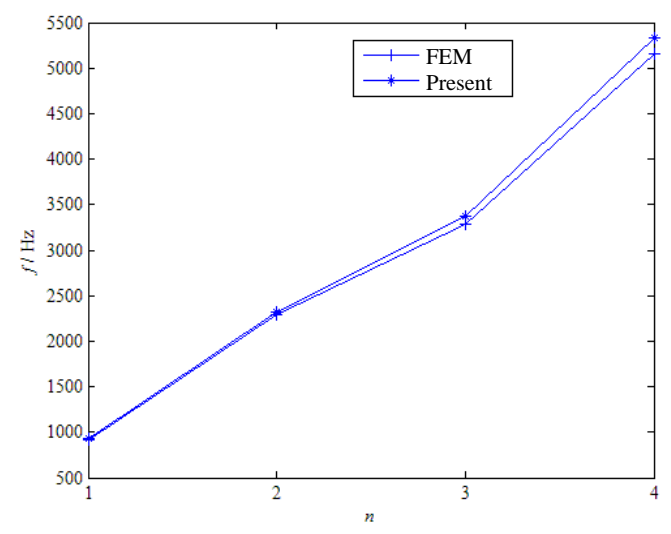

Fig.2 Comparison of the nature frequency with the FEM result 


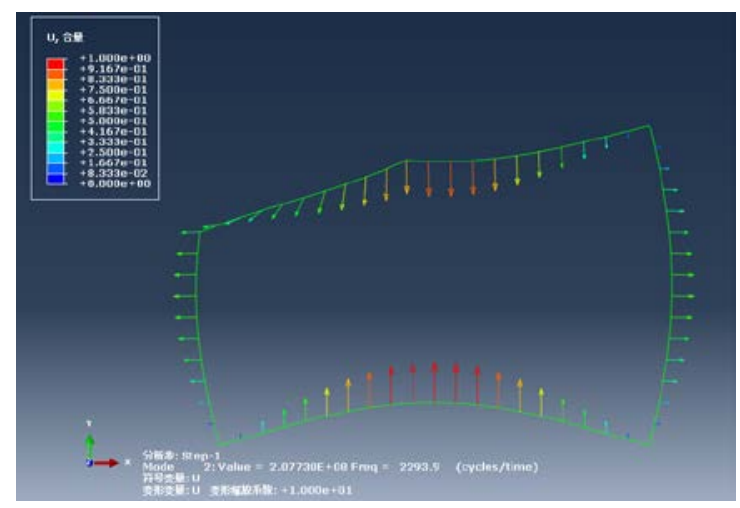

Fig.3 The second modal shape by FEM

Example 3 Considering the PCLD cavity shown in Fig.1, a uniform harmonic excitation $1 e^{i \omega t} \mathrm{~N} / \mathrm{m}$ is applied on front (beam 2) of the cavity. The shape and parameters of the cavity are the same as Example 2. The PCLD structure is treated at the top (beam 5), and the physical parameters of the VEM and CL are the same as Example 1. Setting $h_{2}=h_{3}=0.002 \mathrm{~m}$ and $f=1000 \mathrm{~Hz}$, the displacements of the 3 beam are calculated with and without PCLD treatment, as shown in Fig.4.

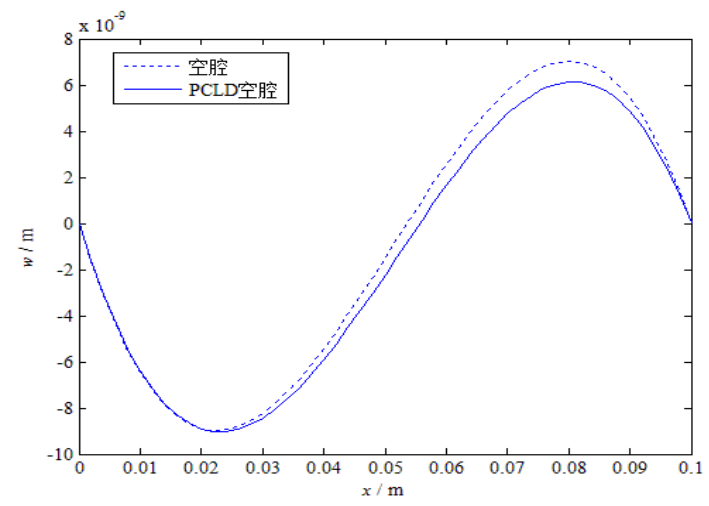

Fig.4 Comparison of the displacement of the cavity

It can be seen from the figure that the displacement of the cavity is reduced with PCLD treatment, which proves that PCLD treatment is of better damping effect. Because the PCLD treatment is covered in the back of the cavity, the damping effect is more obvious at the rear of the beam. Therefore, the position of the PCLD structure should be optimized according to the design requirements of the cavity.

Setting the thickness of the CL $h_{3}=0.002 \mathrm{~m}$, and changing the thickness of the VEM, we calculate and compare the dimensionless displacement ${ }^{\bar{w}}$ of the point $x=0.003 \mathrm{~m}$ from the origin. As shown in Fig5. Setting the thickness of the VEM $h_{2}=0.002 \mathrm{~m}$, and changing the thickness of the CL, the comparison of the frequency response curves is shown in Fig.6. In the figures, the frequency response function is defined as $\mathrm{FRF}=\log _{10}|\bar{w}|$.

It can be seen from the figure that, compared to the cavity base structure $\left(h_{2}=0\right.$ or $\left.h_{3}=0\right)$, the natural frequency of the structure increases in the case of PCLD treatment, while the vibration amplitude decreases. At the low frequencies, the effect of PCLD treatment is very small. As the frequency increases, the damping effect of PCLD treatment increases gradually. It can be seen from Fig.5 that, the vibration reduction at the peak is also enhanced with the increasing of the VEM thickness. As shown from Fig. 6, at the first peak, the response value is the smallest in the case $h_{3}=0.004 \mathrm{~m}$. However, at the second and third peaks, the response in the case $h_{3}=0.004 \mathrm{~m}$ is greater than that of in the case $h_{3}=0.002 \mathrm{~m}$. It can be seen that increasing the thickness of the CL does not necessarily provide a better damping effect, and the size of the PCLD structure needs to be further optimized. 

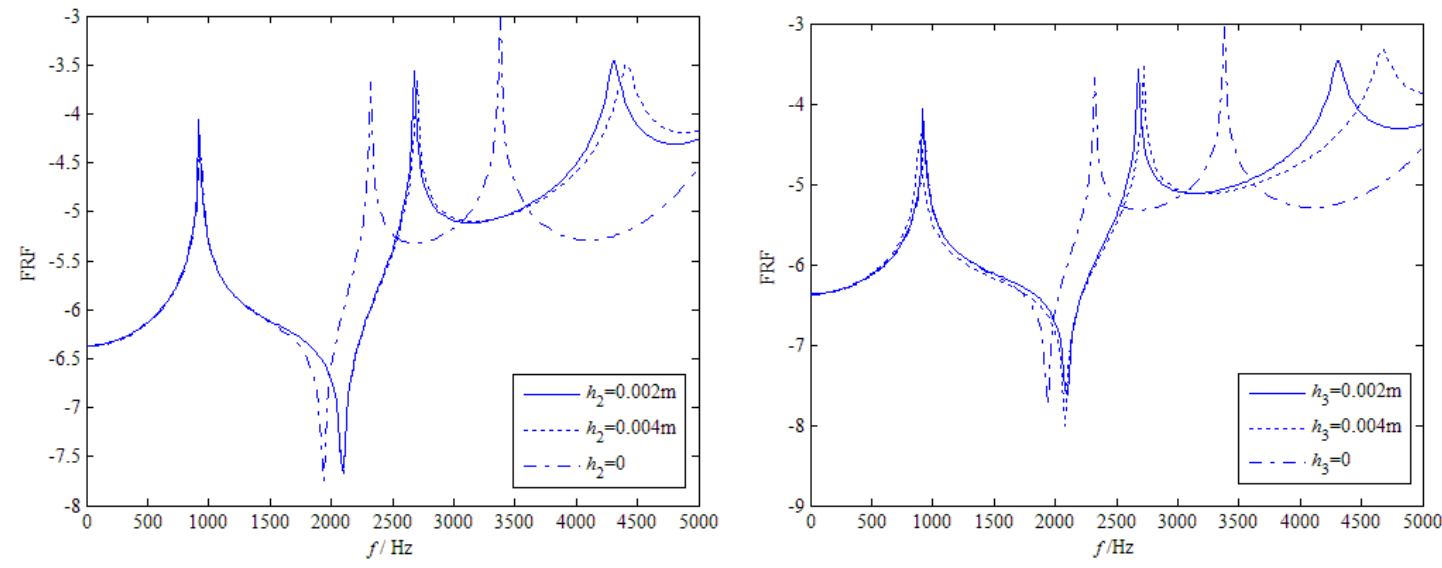

Fig.5 Frequency response function of variation VEM thickness Fig.6 Frequency response function of variation CL thickness

\section{Conclusions}

Based on the dynamic control equations of PCLD beams in the frequency domain, the dynamic model of PCLD beams is established by using the continuity condition and boundary condition at the connection point. Then, a semi-analytical method is proposed to analyze the dynamic characteristics of PCLD cavity by combining the homogeneous expansion precise integration method. The frequency response of the cavity under harmonic excitation is calculated and the influence of structural parameters on vibration and damping characteristics of PCLD cavity is analyzed. The numerical calculations show that the PCLD treatment has a good damping effect in the higher frequency range, and the parameters such as position and thickness have a great influence on the dynamic characteristics of the structure. It is expected that the optimization of the structural parameters can achieve better vibration effect. Comparison with the document and the finite element method results prove that the present method has high precision. Due to the analytic model and high accuracy numerical method, the calculation accuracy and stability can be ensured in the higher frequency range, which can make up the deficiency of finite element method in high frequency range analysis Therefore, the present method can provide a new idea and method for the qualitative vibration analysis and structural optimization of the PCLD cavity.

\section{Acknowledgments}

This work was financially supported by the National Natural Science Foundation of China

(No.51665006, 11502056) fund. Author brief introduction: Lu Jing, Ph.D., professor, mainly engaged in structural vibration and noise control research,E-mail:gxustlj@126.com

\section{References}

[1] Ma Tianfei, LIN Yi, Zhang Jianwei. Morphological analysis of acoustic-solid coupling system in car room [J]. Chinese Journal of Mechanical Engineering, 2005, 41 (7): 225 229.

[2] Liang Zhenwei, Li Yao ming, Zhao Zhan deng, al.Study on local constrained damping of sensor sensitive plate for grain loss monitoring [J]. Journal of Agricultural Mechanization, 2014, 45 (8): $106 \sim 111$.

[3] Shu Gequn, Zhao Wenlong, Liang Xingyu, et al.Study on Vibration Analysis and Structural Parameter Optimization of Constrained Damping Structures [J]. Journal of Xi'an Jiao Tong University, 2014, 48 (3): $108 \sim 114$.

[4] Li Enqi, Lei Yongjun, Tang Guojin et al. Dynamic Analysis of Constrained Layer Damping Beam Based on Transfer Function Method [J]. Journal of Vibration and Shock, 2007, 26 (2): 75 
$\sim 78$.

[5] WANG Ya-yun, LU Jing, YUAN Li-yun et al. Kinetic modeling and analysis of passive constrained layer dampers [J]. Manufacturing Automation, 2014, 36 (12): 53 56,60.

[6] Xiang Yu, Yuan Liyun, Zou Shizhi et al. solving a nonlinear dynamic equations of homogeneous capacity precision integration method [J] Huazhong University of Science and Technology (Natural Science), 2007, 35 (8): 109-111. 